\title{
LINKLESS EMBEDDINGS OF GRAPHS IN 3-SPACE
}

\author{
NEIL ROBERTSON, P. D. SEYMOUR, AND ROBIN THOMAS
}

\begin{abstract}
We announce results about flat (linkless) embeddings of graphs in 3-space. A piecewise-linear embedding of a graph in 3-space is called flat if every circuit of the graph bounds a disk disjoint from the rest of the graph. We have shown:

(i) An embedding is flat if and only if the fundamental group of the complement in 3-space of the embedding of every subgraph is free.

(ii) If two flat embeddings of the same graph are not ambient isotopic, then they differ on a subdivision of $K_{5}$ or $K_{3,3}$.

(iii) Any flat embedding of a graph can be transformed to any other flat embedding of the same graph by "3-switches", an analog of 2-switches from the theory of planar embeddings. In particular, any two flat embeddings of a 4-connected graph are either ambient isotopic, or one is ambient isotopic to a mirror image of the other.

(iv) A graph has a flat embedding if and only if it has no minor isomorphic to one of seven specified graphs. These are the graphs that can be obtained from $K_{6}$ by means of $Y \Delta$ - and $\Delta Y$-exchanges.
\end{abstract}

\section{INTRODUCTION}

All spatial embeddings are assumed to be piecewise linear. If $C, C^{\prime}$ are disjoint simple closed curves in $S^{3}$, then their linking number, $\operatorname{lk}\left(C, C^{\prime}\right)$, is the number of times $(\bmod 2)$ that $C$ crosses over $C^{\prime}$ in a regular projection of $C \cup C^{\prime}$. In this paper graphs are finite, undirected, and may have loops and multiple edges. Every graph is regarded as a topological space in the obvious way. We say that an embedding of a graph $G$ in $S^{3}$ is linkless if every two disjoint circuits of $G$ have zero linking number. The following is a result of Sachs [13, 14] and Conway and Gordon [3].

(1.1) The graph $K_{6}$ (the complete graph on six vertices) has no linkless embedding.

Proof. Let $\phi$ be an embedding of $K_{6}$ into $S^{3}$. By studying the effect of a crossing change in a regular projection, it is easy to see that the mod 2 sum $\sum \operatorname{lk}\left(\phi\left(C_{1}\right), \phi\left(C_{2}\right)\right)$, where the sum is taken over all unordered pairs of disjoint circuits $C_{1}, C_{2}$ of $K_{6}$, is an invariant independent of the embedding. By checking an arbitrary embedding we can establish that this invariant equals 1.

Let $G$ be a graph and let $v$ be a vertex of $G$ of valency 3 with distinct neighbors. Let $H$ be obtained from $G$ by deleting $v$ and adding an edge

Received by the editors January 14, 1992 and, in revised form, May 12, 1992.

1991 Mathematics Subject Classification. Primary 05C10, 05C75, 57M05, 57M15, 57M25.

The first author's research was performed under a consulting agreement with Bellcore; he was supported by NSF under Grant No. DMS-8903132 and by ONR under Grant No. N00014-911-J1905. The third author was supported in part by NSF under Grant No. DMS-9103480, and in part by DIMACS Center, Rutgers University, New Brunswick, New Jersey 08903. 
between every pair of neighbors of $v$. We say that $H$ is obtained from $G$ by a $Y \Delta$-exchange and that $G$ is obtained from $H$ by a $\Delta Y$-exchange. The Petersen family is the set of all graphs that can be obtained from $K_{6}$ by means of $Y \Delta$ - and $\Delta Y$-exchanges. There are exactly seven such graphs, one of which is the Petersen graph. Pictures of these graphs can be found in [13-15]. Sachs $[13,14]$ has in fact shown that no member of the Petersen family has a linkless embedding [the argument is similar to the proof of (1.1)] and raised the problem of characterizing linklessly embeddable graphs. A graph is a minor of another if the first can be obtained from a subgraph of the second by contracting edges. It is easy to see that the property of having a linkless embedding is preserved under taking minors, and that led Sachs to conjecture that a graph is linklessly embeddable if and only if it has no minor in the Petersen family. We have shown that this is true. Moreover, let us say that an embedding $\phi$ of a graph $G$ in $S^{3}$ is flat if for every circuit $C$ of $G$ there exists an open disk in $S^{3}$ disjoint from $\phi(G)$ whose boundary is $\phi(C)$. Clearly every flat embedding is linkless, but the converse need not hold. However, Böhme [1] and Saran [15] conjectured that a graph has a linkless embedding if and only if it has a flat one. This is also true, for we have shown the following.

\section{(1.2) For a graph $G$, the following are equivalent:}

(i) $G$ has a flat embedding,

(ii) $G$ has a linkless embedding,

(iii) $G$ has no minor in the Petersen family.

There have been a number of other attempts $[8,15,2]$ at proving (iii) $\Rightarrow$ (i) and (iii) $\Rightarrow$ (ii). However, none of them is correct.

For the proof of (1.2) we need the following two theorems, which may be of independent interest.

(1.3) Let $\phi$ be an embedding of a graph $G$ in $S^{3}$. Then $\phi$ is flat if and only if for every subgraph $G^{\prime}$ of $G$, the fundamental group of $S^{3}-\phi\left(G^{\prime}\right)$ is free.

Let $\phi_{1}, \phi_{2}$ be two embeddings of a graph $G$ in $S^{3}$. We say that $\phi_{1}, \phi_{2}$ are ambient isotopic if there exists an orientation preserving homeomorphism $h$ of $S^{3}$ onto $S^{3}$ such that $\phi_{1}=h \phi_{2}$. (We remark that by a result of Fisher [4] $h$ can be realized by an ambient isotopy.) If $\phi$ is an embedding of a graph $G$ in $S^{3}$ we denote by $-\phi$ the embedding of $G$ obtained by composing $\phi$ with the antipodal map.

(1.4) Let $G$ be a 4-connected graph and let $\phi_{1}, \phi_{2}$ be two flat embeddings of $G$. Then $\phi_{1}$ is ambient isotopic to either $\phi_{2}$ or $-\phi_{2}$.

\section{THE FUNDAMENTAL GROUP}

A basic tool for working with flat embeddings is the following lemma of Böhme [1] (see also [15]).

(2.1) Let $\phi$ be a flat embedding of a graph $G$ into $S^{3}$, and let $C_{1}, C_{2}, \ldots, C_{n}$ be a family of circuits of $G$ such that for every $i \neq j$, the intersection of $C_{i}$ and $C_{j}$ is either connected or null. Then there exist pairwise disjoint open disks $D_{1}, D_{2}, \ldots, D_{n}$, disjoint from $\phi(G)$ and such that $\phi\left(C_{i}\right)$ is the boundary of $D_{i}$ for $i=1,2, \ldots, n$. 
We illustrate the use of (2.1) with the following, which is a special case of a theorem of $\mathrm{Wu}$ [18]. An embedding $\phi$ of a graph $G$ in $S^{3}$ is spherical if there exists a surface $\Sigma \subseteq S^{3}$ homeomorphic to $S^{2}$ such that $\phi(G) \subseteq \Sigma$. Clearly if $\phi$ is spherical then $G$ is planar.

(2.2) Let $\phi$ be an embedding of a planar graph $G$ in $S^{3}$. Then $\phi$ is flat if and only if it is spherical.

Proof. Clearly if $\phi$ is spherical then it is flat. We prove the converse only for the case when $G$ is 3-connected. Let $C_{1}, C_{2}, \ldots, C_{n}$ be the collection of face-boundaries in some planar embedding of $G$. These circuits satisfy the hypothesis of (2.1). Let $D_{1}, D_{2}, \ldots, D_{n}$ be the disks as in $(2.1)$; then $\phi(G) \cup D_{1} \cup D_{2} \cup \cdots \cup D_{n}$ is the desired sphere.

The following is a result of Scharlemann and Thompson [16].

(2.3) Let $\phi$ be an embedding of a graph $G$ in $S^{3}$. Then $\phi$ is spherical if and only if

(i) $G$ is planar, and

(ii) for every subgraph $G^{\prime}$ of $G$, the fundamental group of $S^{3}-\phi\left(G^{\prime}\right)$ is free.

We see that by (2.2), (1.3) is a generalization of (2.3). In fact, we prove (1.3) by reducing it to planar graphs and then applying (2.3). Let us prove the "only if" part of (1.3). Let $G^{\prime}$ be a subgraph of $G$ such that $\pi_{1}\left(S^{3}-\phi\left(G^{\prime}\right)\right.$ ) is not free. Choose a maximal forest $F$ of $G^{\prime}$ and let $G^{\prime \prime}$ be obtained from $G^{\prime}$ by contracting all edges of $F$, and let $\phi^{\prime \prime}$ be the induced embedding of $G^{\prime \prime}$. Then $\pi_{1}\left(S^{3}-\phi^{\prime \prime}\left(G^{\prime \prime}\right)\right)=\pi_{1}\left(S^{3}-\phi\left(G^{\prime}\right)\right)$ is not free, but $G^{\prime \prime}$ is planar, and so $\phi^{\prime \prime}$ is not flat by (2.2) and (2.3). Hence $\phi$ is not flat, as desired.

Let $G$ be a graph, and let $e$ be an edge of $G$. We denote by $G \backslash e(G / e)$ the graph obtained from $G$ by deleting (contracting) $e$. If $\phi$ is an embedding of $G$ in $S^{3}$, then it induces embeddings of $G \backslash e$ and (up to ambient isotopy) of $G / e$ in the obvious way. We denote these embeddings by $\phi \backslash e$ and $\phi / e$, respectively.

(2.4) Let $\phi$ be an embedding of a graph $G$ into $S^{3}$, and let $e$ be a nonloop edge of $G$. If both $\phi \backslash e$ and $\phi / e$ are flat, then $\phi$ is flat.

Proof. Suppose that $\phi$ is not flat. By (1.3) there exists a subgraph $G^{\prime}$ of $G$ such that $\pi_{1}\left(S^{3}-\phi\left(G^{\prime}\right)\right)$ is not free. If $e \notin E\left(G^{\prime}\right)$ then $\phi \backslash e$ is not flat by (1.3). If $e \in E\left(G^{\prime}\right)$ then $\phi / e$ is not flat by $(1.3)$, because $\pi_{1}\left(S^{3}-(\phi / e)\left(G^{\prime} / e\right)\right)=$ $\pi_{1}\left(S^{3}-\phi\left(G^{\prime}\right)\right)$ is not free.

We say that a graph $G$ is a coforest if every edge of $G$ is a loop. The following follows immediately from (2.4).

(2.5) Let $\phi$ be an embedding of a graph $G$ in $S^{3}$. Then $\phi$ is flat if and only if the induced embedding of every coforest minor of $G$ is flat.

\section{UNIQUENESS}

A graph $H$ is a subdivision of a graph $G$ if $H$ can be obtained from $G$ by replacing edges by pairwise internally-disjoint paths. We recall that Kuratowski's theorem [6] states that a graph is planar if and only if it contains no 
subgraph isomorphic to a subdivision of $K_{5}$ or $K_{3,3}$. It follows from a theorem of Mason [7] and (2.2) that any two flat embeddings of a planar graph are ambient isotopic. On the other hand we have the following.

(3.1) The graphs $K_{5}$ and $K_{3,3}$ have exactly two nonambient isotopic flat embeddings.

Sketch of proof. Let $G$ be $K_{3,3}$ or $K_{5}$, let $e$ be an edge of $G$, and let $H$ be $G \backslash e$. Notice that $H$ is planar. From (2.1) it follows that if $\phi$ is a flat embedding of $G$, then there is an embedded 2-sphere $\Sigma \subseteq S^{3}$ with $\phi(G) \cap \Sigma=$ $\phi(H)$. If $\phi_{1}$ and $\phi_{2}$ are flat embeddings of $G$, we may assume (by replacing $\phi_{2}$ by an ambient isotopic embedding) that this 2 -sphere $\Sigma$ is the same for both $\phi_{1}$ and $\phi_{2}$. Now $\phi_{1}$ is ambient isotopic to $\phi_{2}$ if and only if $\phi_{1}(e)$ and $\phi_{2}(e)$ belong to the same component of $S^{3}-\Sigma$.

As a curiosity we deduce that a graph has a unique flat embedding if and only if it is planar.

We need the following three lemmas. We denote by $f \mid X$ the restriction of a mapping $f$ to a set $X$.

(3.2) Let $\phi_{1}, \phi_{2}$ be two flat embeddings of a graph $G$ that are not ambient isotopic. Then there exists a subgraph $H$ of $G$ isomorphic to a subdivision of $K_{5}$ or $K_{3,3}$ for which $\phi_{1} \mid H$ and $\phi_{2} \mid H$ are not ambient isotopic.

We denote the vertex-set and edge-set of a graph $G$ by $V(G)$ and $E(G)$ respectively. Let $G$ be a graph and let $H_{1}, H_{2}$ be subgraphs of $G$ isomorphic to subdivisions of $K_{5}$ or $K_{3,3}$. We say that $H_{1}$ and $H_{2}$ are 1-adjacent if there exist $i \in\{1,2\}$ and a path $P$ in $G$ such that $P$ has only its endvertices in common with $H_{i}$ and such that $H_{3-i}$ is a subgraph of the graph obtained from $H_{i}$ by adding $P$. We say that $H_{1}$ and $H_{2}$ are 2-adjacent if there are seven vertices $u_{1}, u_{2}, \ldots, u_{7}$ of $G$ and thirteen paths $L_{i j}$ of $G(1 \leq i \leq 4$ and $5 \leq j \leq 7$, or $i=3$ and $j=4)$, such that

(i) each path $L_{i j}$ has ends $u_{i}, u_{j}$,

(ii) the paths $L_{i j}$ are mutually vertex-disjoint except for their ends,

(iii) $H_{1}$ is the union of $L_{i j}$ for $i=2,3,4$ and $j=5,6,7$, and

(iv) $H_{2}$ is the union of $L_{i j}$ for $i=1,3,4$ and $j=5,6,7$.

(Notice that if $H_{1}$ and $H_{2}$ are 2-adjacent then they are both isomorphic to subdivisions of $K_{3,3}$ and that $L_{34}$ is used in neither $H_{1}$ nor $H_{2}$.) We denote by $\mathscr{K}(G)$ the simple graph with vertex-set all subgraphs of $G$ isomorphic to subdivisions of $K_{5}$ or $K_{3,3}$ in which two distinct vertices are adjacent if they are either 1-adjacent or 2-adjacent. The following is easy to see, using (3.1).

(3.3) Let $\phi_{1}, \phi_{2}$ be two flat embeddings of a graph $G$, and let $H, H^{\prime}$ be two adjacent vertices of $\mathscr{K}(G)$. If $\phi_{1} \mid H$ is ambient isotopic to $\phi_{2} \mid H$, then $\phi_{1} \mid H^{\prime}$ is ambient isotopic to $\phi_{2} \mid H^{\prime}$.

The third lemma is purely graph-theoretic.

(3.4) If $G$ is a 4-connected graph, then $\mathscr{K}(G)$ is connected.

We prove (3.4) in [10] by proving a stronger result, a necessary and sufficient condition for $H, H^{\prime} \in V(\mathscr{K}(G))$ to belong to the same component of $\mathscr{K}(G)$ in an arbitrary graph $G$. The advantage of this approach is that it permits an inductive proof using the techniques of deleting and contracting edges. 
Proof of (1.4). If $G$ is planar then $\phi_{1}$ is ambient isotopic to $\phi_{2}$ by Mason's theorem. Otherwise there exists, by Kuratowski's theorem, a subgraph $H$ of $G$ isomorphic to a subdivision of $K_{5}$ or $K_{3,3}$. By replacing $\phi_{2}$ by $-\phi_{2}$ we may assume by (3.1) that $\phi_{1} \mid H$ is ambient isotopic to $\phi_{2} \mid H$. From (3.3) and (3.4) we deduce that $\phi_{1} \mid H^{\prime}$ is ambient isotopic to $\phi_{2} \mid H^{\prime}$ for every $H^{\prime} \in V(\mathscr{K}(G))$. By (3.2) $\phi_{1}$ and $\phi_{2}$ are ambient isotopic, as desired.

We now state a generalization of (1.4). Let $\phi$ be a flat embedding of a graph $G$, and let $\Sigma \subseteq S^{3}$ be a surface homeomorphic to $S^{2}$ meeting $\phi(G)$ in a set $A$ containing at most three points. In one of the open balls into which $\Sigma$ divides $S^{3}$, say $B$, choose an open disk $D$ with boundary a simple closed curve $\partial D$ such that $A \subseteq \partial D \subseteq \Sigma$. Let $\phi^{\prime}$ be an embedding obtained from $\phi$ by taking a reflection of $\phi$ through $D$ in $B$ and leaving $\phi$ unchanged in $\Sigma-B$. We say that $\phi^{\prime}$ is obtained from $\phi$ by a 3-switch. The following analog of a theorem of Whitney [17] generalizes (1.4).

(3.5) Let $\phi_{1}, \phi_{2}$ be two flat embeddings of a graph $G$ in $S^{3}$. Then $\phi_{2}$ can be obtained from $\phi_{1}$ by a series of 3-switches.

\section{MAIN THEOREM}

The difficult part of (1.2) is to show that (iii) implies (i). Let us just very briefly sketch the main idea of the proof. Suppose that $G$ is a minor-minimal graph with no flat embedding. We first show that a $Y \Delta$-exchange preserves the property of having a flat embedding; thus we may assume that $G$ has no triangles (and indeed has some further properties that we shall not specify here). It can be shown that $G$ satisfies a certain weaker form of 5-connectivity. Suppose that there are two edges $e, f$ of $G$ so that $G \backslash e / f$ and $G / e / f$ are "Kuratowski 4-connected". (Kuratowski 4-connectivity is a slight weakening of 4connectivity for which (1.4) still remains true.) Since $G$ is minor-minimal with no flat embedding, there are flat embeddings $\phi_{1}, \phi_{2}, \phi_{3}$ of $G \backslash e, G / e, G / f$, respectively. Since $G \backslash e / f$ and $G / e / f$ are both Kuratowski 4-connected, we can assume (by replacing $\phi_{1}$ or $\phi_{2}$ or both by its mirror image) that $\phi_{1} / f$ is ambient isotopic to $\phi_{3} \backslash e$ and that $\phi_{2} / f$ is ambient isotopic to $\phi_{3} / e$. Now it can be argued (the details are quite complicated, see [12]) that the uncontraction of $f$ in $\phi_{1} / f \simeq \phi_{3} \backslash e$ is the same as in $\phi_{2} / f \simeq \phi_{3} / e$. Let $\phi$ be obtained from $\phi_{3}$ by doing this uncontraction; then $\phi \backslash e$ is ambient isotopic to $\phi_{1}$ and $\phi / e$ is ambient isotopic to $\phi_{2}$. Since both these embeddings are flat, $\phi$ is flat by (2.4), a contradiction. Thus no two such edges $e, f$ exist. But now a purely graph-theoretic argument [11] (using the nonexistence of such edges $e, f$, the high connectivity of $G$, and that the graph obtained from $G$ by deleting $v$ is nonplanar for every vertex $v$ of $G$ ) implies $G$ has a minor in the Petersen family.

Finally we would like to mention some algorithmic aspects of flat embeddings. In [16] Scharlemann and Thompson describe an algorithm to test if a given embedding is spherical. Using their algorithm, (2.2), and (2.5), we can test if a given embedding is flat, by testing the flatness of all coforest minors. At the moment there is no known polynomial-time algorithm to test if an embedding of a given coforest is flat, because it includes testing if a knot is trivial. On the other hand, we can test in time $O\left(|V(G)|^{3}\right)$ if a given graph $G$ has a flat embedding. 
This is done by testing the absence of minors isomorphic to members of the Petersen family, using the algorithm [9] of the first two authors.

\section{REFERENCES}

1. T. Böhme, On spatial representations of graphs, Contemporary Methods in Graph Theory (R. Bodendieck, ed.), Mannheim, Wien, Zurich, 1990, pp. 151-167.

2. L Lecture at the AMS Summer Research Conference on Graph Minors, Seattle, WA, June 1991.

3. J. H. Conway and C. McA. Gordon, Knots and links in spatial graphs, J. Graph Theory 7 (1983), 445-453.

4. G. M. Fisher, On the group of all homeomorphisms of a manifold, Trans. Amer. Math. Soc. 97 (1960), 193-212.

5. D. W. Hall, A note on primitive skew curves, Bull. Amer. Math. Soc. 49 (1943), 935-937.

6. C. Kuratowski, Sur le problème des courbes gauches en topologie, Fund. Math. 15 (1930), 271-283.

7. W. K. Mason, Homeomorphic continuous curves in 2-space are isotopic in 3-space, Trans. Amer. Math. Soc. 142 (1969), 269-290.

8. R. Motwani, A. Raghunathan, and H. Saran, Constructive results from graph minors: Linkless embeddings, Proc. 29th Symposium on the Foundations of Computer Science, Yorktown Heights, 1988.

9. N. Robertson and P. D. Seymour, Graph minors. XIII. The disjoint paths problem, submitted.

10. N. Robertson, P. D. Seymour, and R. Thomas, Kuratowski chains, submitted.

11. __ Petersen family minors, submitted.

12. __ Sachs' linkless embedding conjecture, manuscript.

13. H. Sachs, On spatial representation of finite graphs (Proceedings of a conference held in Lagów, February 10-13, 1981, Poland), Lecture Notes in Math., vol. 1018, Springer-Verlag, Berlin, Heidelberg, New York, and Tokyo, 1983.

14. _ On spatial representations of finite graphs, finite and infinite sets, (A. Hajnal, L. Lovász, and V. T. Sós, eds), Colloq. Math. Soc. János Bolyai, vol. 37, North-Holland, Budapest, 1984, pp. 649-662.

15. H. Saran, Constructive results in graph minors: Linkless embeddings, Ph.D. thesis, University of California at Berkeley, 1989.

16. M. Scharlemann and A. Thompson, Detecting unknotted graphs in 3-space, J. Differential Geom. 34 (1991), 539-560.

17. H. Whitney, 2-isomorphic graphs, Amer. J. Math. 55 (1933), 245-254.

18. Y.-Q. Wu, On planarity of graphs in 3-manifolds, Comment. Math. Helv. (to appear).

Department of Mathematics, Ohio State University, 231 West 18th Avenue, Columbus, OHIO 43210

E-mail address: robertso@function.mps.ohio-state.edu

Bellcore, 445 South Street, Morristown, New Jersey 07962

E-mail address: pds@bellcore.com

School of Mathematics, Georgia Institute of Technology, Atlanta, Georgia 30332

E-mail address: thomas@math.gatech.edu 